09 , and dramatic growth since, reaching 15,000 to 33,000 in 2018 (41 to 90 per day).

Conclusions More than 40 SRs are now being published daily and stakeholders need to be more aware of Epistemonikos as an aggregating resource. Although the platform might not provide access to all SRs found by a full literature search it would augment such searches and is likely to be adequate in resource-limited situations. If we cannot keep up with SR output when the challenge is four to eight times greater than a decade ago, ways need to be found to focus on high quality SRs that will provide a valid base for EBM, support the increased systematic use of existing evidence and reduce waste.

\section{A SYSTEMATIC REVIEW AND TAXONOMY OF ASSESSMENT TOOLS FOR EVALUATING EDUCATION IN EVIDENCE-BASED MEDICINE}

${ }^{1}$ Bharathy Kumaravel, ${ }^{2}$ Jasmine Hearn, ${ }^{1}$ Rachel Pollard, ${ }^{1}$ Claire Stocker, ${ }^{3}$ David Nunan. ${ }^{1}$ University of Buckingham Medical School, Buckingham, UK; ${ }^{2}$ Manchester Metropolitan University, Manchester, UK; ${ }^{3}$ Centre for Evidence Based Medicine, Oxford, UK

\subsection{6/bmjebm-2019-EBMLive.107}

Objectives The importance of teaching the skills and practice of Evidence-Based Medicine (EBM) for medical professionals has steadily grown in recent years. Alongside this growth is a need to evaluate the effectiveness of EBM curriculum on learners' knowledge, skill, attitudes, competency and behaviour. A previous 2006 systematic review identified published evaluation instruments and tools focused predominantly knowledge, skills and attitudes, and few had been formally validated. There have been a number of tools published since the 2006 review, and at present there is no taxonomy of existing tools to aid EBM educators. The aim of this systematic review was to provide an up-to-date taxonomy and appraisal of assessment instruments that purport to evaluate learners' EBM knowledge, skills, attitudes, competency and behaviour.

Method We searched MEDLINE, EMBASE, Cochrane library, Educational Resources Information Centre (ERIC), Best Evidence Medical Education (BEME) databases and references of retrieved articles published between January 2005 and March 2019 for assessment tools used to evaluate EBM teaching at any stage in medical education. Two reviewers independently performed data extraction and quality assessment. Quantitative and qualitative data on the development and description of the tool, number of participants, training level of participants, EBM domain(s) evaluated, level(s) of educational evaluation addressed, psychometric properties and feasibility were extracted. The quality of tools was assessed by the number of domains of EBM assessed, levels of educational evaluation addressed, robustness of psychometric testing and reporting of feasibility.

Results 155 of 1608 potentially relevant articles were assessed for full text review following title and abstract screening. Of these, 11 articles describing seven unique instruments met predefined criteria for inclusion. Together with those previously identified $[\mathrm{n}=2]$, this presents a total of seven instruments for evaluation of EBM teaching in medical education. Level 1 tests such as the educational prescriptions (EP) and Assessing Competency in EBM (ACE) addressed at least three domains of EBM, two levels of educational evaluation, reported good discriminatory ability and feasibility $(n=2)$. The Fresno, Berlin, EBM test and Objective Structured Clinical Examination
(OSCE) were categorised as level $2(n=4)$. The Biostatistics and Clinical Epidemiology Skills Assessment (BACES) addressed just one domain, two levels, reported no psychometric properties or feasibility and was categorised as level 3 $(n=1)$. Few instruments evaluated the application of EBM skills either in a simulated case scenario or in real clinical cases.

Conclusions We report our interim findings from this ongoing systematic review. Our review captured an additional five instruments, bringing the total of available instruments to seven. The majority (86\%) of these has reasonable validity. Our review has identified educational prescriptions (EP) and ACE as level 1 tools; Fresno, Berlin, EBM test and OSCE are level 2 tools and BACES as level 3. Further development and validation of assessment tools that evaluate all the steps in EBM is needed. The findings from this systematic review will facilitate medical educators by offering a taxonomy of assessment tools to aid them with evaluation of their teaching and learning.

\section{ARE ALL RESEARCHES ADEQUATELY RESEARCHED?}

Shabana Ali. Practicing Clinician; Ex-Student of Terna Medical College, MUMBAl, India

\subsection{6/bmjebm-2019-EBMLive.108}

Misrepresentation of medical-research by mass-media is common as thousands of medical journals compete for attention. 'Brushing teeth twice a day could prevent erectile dysfunction while bad oral hygiene may triple the risk of impotency!' read a newspaper headline. In this example, distortion of research-results is probably an effect of poor understanding of relative-risk, confounding and/or interactions. Anyone can be a publisher read by millions of followers; bypassing professional editors and peer-review from submission process, thus, leaving presented outcomes and methods unchallenged. Ability to prove results distinguishes blogger from researcher and expert. The 'crisis of expertise' affects clinical research which is often undermined by underpowered design, poor reproducibility of chosen methods, extrapolation and over-interpretation of data, under-reporting of negative results and publication-bias. Transparency and Accountability are key to good research. Reporting of 'conflicts of interest' is associated with involvement of pharmaceutical companies and policymaking bodies as suggested by BMJ publications in 'the weekend effect on mortality'. After Lancet retracted article linking autism to MMR vaccines, citing 'public interest in the issue', it was apparent to the wider research community that even most respected journals and institutions are not immune to fraudulent research. It's concerning that this case was exposed as a result of journalistic investigation, rather than academic-vigilance. Unfortunately, even Randomised-Controlled Trials (RCTs) can be affected by potential biases of poor-design or selection-bias. The peer-review process serves as a great filter to improve research standards. Improving selection, incentives and comprehensive training on methodology \& statistics of those involved in research-process may help in execution of high scientific standards and reporting of clinical research. Systematic-reviews should take precedence over just-experience. Data-monitoring committees and independent expert-panels reviewing trial-data regularly ensure RCT-trial integrity. However, standards and competencies of these 
bodies are not universal. Global platforms like EQUATOR (Enhancing the QUAlity and Transparency Of health Research) Network help to enhance the reporting standards and credibility of research articles. New Systematic-review data-repositories will allow access to raw data for early research-comparison. 'Artificial Intelligence' software with automatic-machine-learning-algorithms can filter flawed researches from genuine ones by identifying outliers early. The rising competition between journals and media's insatiable hunger for newer scientific discoveries has laid a lot of pressure on the researchers. Media sensationalism should be resisted to avoid uncertainties and reduce pressure over scientists. Government funding agencies and Universities can pull the brakes to statistical malpractices by cutting-off support and rewards for trials with flawed structures. The cornerstones of evidence-based medicine rely on adequateappraisal, systematic-review and authenticating-evidence.

Conclusions A good clinical study must be non-biased, with a core of ethics and laced with scientific advancements. Peer-review, Refined Reporting-standards, Raw-data publication in open-access journals, Systematic-review repositories, Data-synthesis and comparison, Independent-funders, multisite studies and limiting conflicts of interests can safeguard rational research. Not all researchers or funding-bodies intend to fraud. But, too many temptations along the route incite them to indulge in scientific-misconduct which is a critical waste of funds. It is, therefore, essential to promote the agenda of 'Transparency-or-Perish' rather 'Publish-orPerish'!

\section{IN THE AGE OF GOOGLE, PATIENTS ARE TAKING ON MORE ACTIVE ROLES IN THEIR MEDICAL CARE, AND THE PHYSICIAN'S WORD IS NO LONGER SEEN AS GOSPEL}

Victoria McKinnon. MMaster University, Hamilton, Canada

10.1136/bmjebm-2019-EBMLive.109

As a result, the medical community has witnessed increasing numbers of patients turn from Western medicine towards alternative healthcare. While some professionals scoff at this shift, in many ways it is the logical result of exposing a nonmedical community to the overwhelming, and, at times, misleading, body of evidence that supports Western medical practices. One particularly egregious example of this shift is the rise of anti-vaccination sentiment. Many anti-vaxxer parents have invested their time in understanding the 'science' that backs such sentiment. This leads one to believe that it is not a decreasing societal intelligence or lack of interest that has resulted in a movement away from modern medicine; rather, the shift is more likely attributable to the accessibility of alternative medicine texts as compared to scientific research. Medical research at best makes sense to the medical community; at worst, only those with niche training in the topic understand it. As such, how can we expect findings to translate over adequately to the patient community? If we wish to make research relevant to patients, and if we hope to ensure physicians with limited statistical backgrounds are applying new research appropriately, then it is required that we develop a system whereby an outside party conveys study results clearly, succinctly, and with minimised bias. I propose that this is best accomplished through the widespread integration of lay summaries alongside study abstracts. A lay summary is essentially a précis that has been written in layperson's terms, allowing those without a background in the subject to obtain an accurate understanding of it. Lay summaries are already used in the context of grant committees, as patient opinion is considered a critical component of clinical research funding. They have also been shown to benefit medical professionals on the committees with differing areas of expertise. The summary itself should be written by an outside body, ideally a statistician, who can maintain some level of objectivity and reliability in the reporting of results. Another benefit of using an outside reviewer is that it holds the authors accountable to the robustness of their methods; knowing that someone with an expertise in statistical analysis will be reviewing the study and summarising the results adds a certain level of expectation and pressure for the analysis to be performed well. As with abstracts, the lay summary should be made publicly available. Thus, even if patients do not have access to the study, they can still obtain a fundamental understanding of the available evidence and its statistical strength. The major barrier for this idea is the cost of the statistician. However, I would argue that the addition of lay summaries could broaden a journal's readership and strengthen its reputation as a reputable source of knowledge, thereby redeeming any monetary losses while simultaneously increasing accessibility, reducing bias, and minimising questionable research practices. 